\title{
PERÚ, CAMBIO CLIMÁTICO Y ENFERMEDADES NO TRASMISIBLES: ¿DÓNDE ESTAMOS Y A DÓNDE VAMOS?
}

\begin{abstract}
José L. Avilez ${ }^{1,2, a}$, Janina Bazalar,b, Diego Azañedo ${ }^{3, c}$, J. Jaime Miranda ${ }^{1,2, d}$
\section{RESUMEN}

El mundo atraviesa una crisis de enfermedades no transmisibles (ENT) junto con un panorama de volatilidad climática. El 99\% de la carga en salud del cambio climático será asumida por los países en vías de desarrollo. La mejor oportunidad que tiene el Perú frente a la inminencia del cambio climático recae en desarrollar políticas enfocadas en crear cobeneficios mediante las estrategias de mitigación y adaptación al cambio climático que, a la vez, representen mejoras sobre el panorama de las ENT y la salud en general. En este artículo presentamos los compromisos asumidos por el Perú en estrategias de mitigación de emisiones de gases de efecto invernadero (GEI); identificamos oportunidades en salud pública para el Perú en el Acuerdo de París de la 21. ${ }^{\text {a }}$ Conferencia de las Partes (COP21); y culminamos con las recomendaciones sobre la generación de evidencia y políticas para para la Academia y el Gobierno.
\end{abstract}

Palabras Clave: Cambio climático; Enfermedad crónica; Política de salud; Perú (DeCS BIREME)

\section{PERU, CLIMATE CHANGE AND NON-COMMUNICABLE DISEASES: ¿WHERE ARE WE AND WHERE ARE WE HEADED?}

\begin{abstract}
The world is facing a crisis of noncommunicable diseases alongside a state of climate volatility. Of the total health burden attributable to climate change, $99 \%$ will be assumed by developing countries. In light of climate change, Peru's best opportunity is to create mitigation and adaptation policies focused on developing health co-benefits, which will improve the state of noncommunicable diseases and the climate system. This article presents Peru's compromises in terms of mitigation of greenhouse gas emissions; identifies public health opportunities for Peru under the Paris Agreement signed in the 21st Conference of the Parties (COP21); and makes recommendations for evidence and policy generation for the National Academy of Medicine and the Peruvian government.
\end{abstract}

Key Words: Climate change; Chronic disease; Health policy; Peru (MeSH NLM)

\section{INTRODUCCIÓN}

Vivimos en un mundo donde el $99 \%$ de la carga en salud del cambio climático será impuesta en países en vías de desarrollo, a pesar de que producen el $3 \%$ de las emisiones de carbono ${ }^{(1)}$. Este vínculo requiere ser abordado, comprendido y difundido por los profesionales de salud. En este artículo, iniciamos con una revisión del estado actual del cambio climático y salud con énfasis en enfermedades crónicas no trasmisibles (ENT); presentamos los compromisos del Perú en estrategias de mitigación de emisiones de gases de efecto invernadero (GEI); identificamos oportunidades en salud pública para el Perú en el Acuerdo de la 21. ${ }^{a}$ Conferencia de las Partes (COP21); y culminamos con las recomendaciones de generación de evidencia y políticas para la Academia Nacional de Medicina y el gobierno.

\section{EL CAMBIO CLIMÁTICO Y LAS ENFERMEDADES NO TRASMISIBLES: STATUS QUO}

El mundo atraviesa una crisis de ENT, volatilidad climática e inestabilidad del desarrollo económico. La magnitud de la crisis es tal, que ambos, las ENT (2) y el cambio climático (3), son considerados las mayores amenazas para la salud del siglo XXI. El Grupo Intergubernamental de Expertos sobre el Cambio Climático (Intergovernmental Panel on Climate Change, IPCC) define al cambio climático como:

\footnotetext{
Facultad de Medicina, Universidad Peruana Cayetano Heredia. Lima, Perú.

CRONICAS, Centro de Excelencia en Enfermedades Crónicas, Universidad Peruana Cayetano Heredia. Lima, Perú.

Instituto de Investigación, Universidad Católica los Ángeles de Chimbote. Chimbote, Perú.

Estudiante de Medicina; ${ }^{\mathrm{b}}$ licenciada en Enfermería; ${ }^{\mathrm{c}}$ cirujano dentista, ${ }^{\mathrm{d}}$ médico, magíster y doctor en Epidemiología

Recibido: 20-01-16 Aprobado: 09-03-16
} 
Una variación estadística en el estado medio del clima o en su variabilidad, que persiste durante un período prolongado (4). El mundo experimentó un aumento de $0,85{ }^{\circ} \mathrm{C}$ en el período $1880-2012$ y se estima que el aumento en el período 1990-2100 será entre $1,4-4,0^{\circ} \mathrm{C}^{(5)}$.

La evidencia de los efectos del cambio climático sobre la salud es contundente ${ }^{(6-9)}$. Se estimó que para el 2000 , con respecto al período 1961-1990, ya se habían acumulado 5500000 años de vida ajustados por discapacidad debido al cambio climático, equivalente al $0,4 \%$ de la carga mundial de morbilidad. Por lo tanto, la Organización Mundial de la Salud predice que, si los cambios medioambientales no son controlados, sus efectos en la mortalidad y morbilidad pueden incrementar dramáticamente ${ }^{(10)}$.

Por otro lado, las ENT también son una amenaza para la salud; en el 2008 causaron el $63 \%$ de todas las muertes a nivel global, donde predominan enfermedades cardiovasculares, cáncer, enfermedades respiratorias crónicas y diabetes, y podrían causar pérdidas económicas de 15 trillones de dólares en los próximos quince años (2). A pesar de que la Asamblea General de la Organización de Naciones Unidas (ONU) haya declarado que; Las enfermedades no transmisibles constituyen uno de los principales obstáculos para el desarrollo en el siglo XXI (11), la retórica política y las resoluciones de la ONU no han mejorado la conciencia -pública y política- sobre las ENT ${ }^{(12)}$.

A la luz de estos hallazgos, es sorprendente que el vínculo entre las ENT y el cambio climático no haya recibido suficiente atención en los ámbitos de investigación y políticas públicas. No obstante, varios mecanismos se han propuesto como vínculo entre las dos ${ }^{(13,14)}$. Dos de los factores de riesgo modificables para las ENT, actividad física y dietas inadecuadas, comparten una relación estrecha con el cambio climático. Por ejemplo, la urbanización ha creado una relación intrincada entre el cambio climático y las ENT. Los procesos de urbanización vienen acompañados con un aumento del transporte vehicular, mayores emisiones de GEl y mayor contaminación atmosférica; estas ramificaciones van de la mano con un mayor tiempo sentado y una reducción de la actividad física, factores que conllevan aumentos en el índice de masa corporal y en las tasas de obesidad y mortalidad. Además, la contaminación atmosférica por GEl está asociada con la morbilidad por enfermedades cardiovasculares y respiratorias ${ }^{(13)}$. Sobre las dietas inadecuadas, el cambio climático perjudica la calidad del suelo, reduce la producción de agricultura y socava la seguridad alimentaria. La combinación de estos factores, una menor actividad física y peores patrones de alimentación, se traduce en mayor obesidad. Algunas predicciones indican que la obesidad, un problema de salud pública ya establecido, podría estar directamente vinculada con el cambio climático debido a los requerimientos de producción adicional de alimentos, así como las mayores emisiones de GEl vinculadas al transporte de poblaciones con un mayor promedio de peso ${ }^{(15)}$.

A pesar de que se hayan establecido prioridades de investigación (16) y acción (17) en el frente de ENT, no hay guías sobre qué investigar o evidencia sobre qué hacer en el ámbito Clima-ENT. Dado que la mayor carga en salud del cambio climático y ENT será asumida por países en vías de desarrollo ${ }^{(14)}$, es imperativo producir recomendaciones de políticas y guías de investigación para estos países.

Para el Perú -país vulnerable a los efectos del cambio climático que se encuentra en medio de una transición epidemiológica- la COP21 proveyó información valiosa y esclareció la necesidad de trabajar en esta área para producir evidencia, intervenciones y mejoras. En las siguientes secciones exponemos las oportunidades y recomendaciones para generar políticas en el ámbito de salud pública dirigidas a las ENT en el Perú.

\section{EL PERÚ Y LA CONVENCIÓN MARCO DE LAS NACIONES UNIDAS SOBRE EL CAMBIO CLIMÁTICO}

La Convención Marco de las Naciones Unidas sobre el Cambio Climático (CMNUCC) es un instrumento legal vinculante para el control del cambio climático ${ }^{(18)}$. El objetivo último de la Convención es estabilizar las concentraciones atmosféricas de GEI para prevenir la interferencia antropogénica en los sistemas climáticos.

Acorde al párrafo 1 del Artículo 4, el Perú, como parte de la CMNUCC, se comprometió a minimizar y mitigar los efectos adversos del cambio climático en la salud pública. Asimismo, la CMNUCC establece la Conferencia de las Partes (COP), que se reúne cada año para determinar los compromisos de cada Estado frente al objetivo de reducir las emisiones de GEI. En la COP20, celebrada en Lima en el 2014, se firmó el "Llamado de Lima para la Acción Climática", donde resaltó el compromiso de las naciones de presentar las denominadas Contribuciones Previstas y Determinadas a Nivel Nacional (Intended Nationally Determined Contribution, iNDC) donde cada país establece sus planes de mitigación y adaptación (19).

EnlaCOP21 se firmóelAcuerdo de París, cuyo objetivo principal es limitar el calentamiento global a $2^{\circ} \mathrm{C}$. En el preámbulo del Acuerdo, se reconoce el derecho a la salud, particularmente el de poblaciones vulnerables al cambio climático.

El iNDC del Perú (20) señala que nuestro país tiene siete de las nueve características necesarias para clasificar al país como "particularmente vulnerable". El iNDC estima 
que para el 2050, el Perú experimentará un aumento de temperatura de $2{ }^{\circ} \mathrm{C}$ y un aumento en $20 \%$ de la variabilidad de precipitación. Junto con el crecimiento económico de los últimos años, el Perú ha sido capaz de desarrollar proyectos de mitigación del cambio climático con apoyo internacional. En este contexto, el Perú se ha propuesto la meta de reducir sus emisiones de GEI en $30 \%$ para el 2030 , con $20 \%$ provisto de recursos internos públicos y privados y el otro $10 \%$ supeditado al financiamiento externo internacional.

A raíz de los estudios de vulnerabilidad al cambio climático en el Perú, el Ministerio de Ambiente priorizó la adaptación de cinco sistemas para alcanzar el objetivo último de la CMNUCC hacia el año 2030, donde la salud ocupa el quinto lugar. A partir de estas prioridades, en diciembre de 2015 se inició la formulación del Plan de Adaptación Nacional, acorde al Acuerdo de París. El iNDC sobre salud aborda el incremento de la capacidad adaptativa de los servicios de salud para enfrentar al cambio climático mediante el mejoramiento de las cinco funciones del actual Sistema Nacional de Salud: prevención de enfermedades, promoción de la salud, protección de la salud, restauración de la salud y rehabilitación. El objetivo de esta contribución es: Reducir la vulnerabilidad e incrementar la resiliencia de la población ante el efecto del cambio climático en la salud ${ }^{(21)}$.

Desde este punto de partida, el Perú puede tomar dos opciones: políticas de mitigación o adaptación. Las políticas de mitigación reducen el calentamiento global e involucran la reducción de los GEI. Las políticas de adaptación fortalecen la resiliencia y reducen la vulnerabilidad de las personas y sistemas hacia el cambio climático observado y esperado (ver glosario, Tabla 1).

El Perú adoptó una política de mitigación al plantear la reducción de sus emisiones en $30 \%$, cantidad que se mantiene contingente al apoyo internacional que reciba. Queda pendiente adoptar políticas de adaptación, las cuales deberían enfatizar la interfaz Clima-ENT.

En el Acuerdo de París, se resaltó que los países desarrollados deben liderar la lucha contra el cambio climático. El Perú, como país en vías de desarrollo, debe alimentarse de esta posición, particularmente del

Tabla 1. Glosario

\begin{tabular}{|c|c|}
\hline Término & Definición \\
\hline Acuerdo de París & $\begin{array}{l}\text { Es el acuerdo firmado en la COP21 donde se acordó limitar el incremento de la temperatura } \\
\text { media global a } 2{ }^{\circ} \mathrm{C} \text { con respecto a niveles preindustriales y limitar las emisiones globales de } \\
\text { gases de efecto invernadero. El acuerdo entrará en acción a partir del } 2020^{(22)} \text {. }\end{array}$ \\
\hline Adaptación al cambio climático & $\begin{array}{l}\text { Es la adaptación de la sociedad en respuesta a las variaciones climáticas, con el objetivo } \\
\text { de aminorar el daño provocado por estas variaciones }{ }^{(4)} \text {. Adaptación preventiva requiere } \\
\text { implementar medidas para prepararse ante el cambio climático. Adaptación reactiva es } \\
\text { implementar medidas cuando se experimenten los efectos del cambio climático. }\end{array}$ \\
\hline Calentamiento global & $\begin{array}{l}\text { Es el aumento de la temperatura media global de la superficie de la tierra causado por las } \\
\text { emisiones antropogénicas de gases de efecto invernadero }{ }^{(29)} \text {. }\end{array}$ \\
\hline Cambio climático & $\begin{array}{l}\text { Son los cambios en las características climáticas; por ejemplo: temperatura, humedad, lluvia } \\
\text { viento y fenómenos meteorológicos por períodos de tiempo prolongados }{ }^{(30)} \text {. La CMNUCO } \\
\text { considera el cambio climático como "cambio de clima atribuido directa o indirectamente a la } \\
\text { actividad humana que altera la composición de la atmósfera mundial" (18). }\end{array}$ \\
\hline Capacidad de adaptación & $\begin{array}{l}\text { Conjunto de capacidades, recursos e instituciones de un estado o país que permitirán } \\
\text { implementar o tomar medidas de adaptación eficaces para el cambio climático (4). }\end{array}$ \\
\hline Conferencia de las Partes (COP) & $\begin{array}{l}\text { La Conferencia de las Partes es el órgano supremo de la Convención Marco de las Naciones } \\
\text { Unidas sobre el Cambio Climático. Es aquel que examina todo documento o instrumento } \\
\text { jurídico, y quién adopta las decisiones necesarias para promover la aplicación efectiva de la } \\
\text { CMNUCC }{ }^{(18)} \text {. }\end{array}$ \\
\hline $\begin{array}{l}\text { Convención Marco de las Naciones } \\
\text { Unidas sobre el Cambio Climático } \\
\text { (CMNUCC) }\end{array}$ & $\begin{array}{l}\text { Instrumento legal de naturaleza vinculante que establece las obligaciones básicas de todas } \\
\text { las Partes para combatir el cambio climático }{ }^{(18)} \text {. }\end{array}$ \\
\hline $\begin{array}{l}\text { Intended Nationally Determined } \\
\text { Contributions (iNDC) }\end{array}$ & $\begin{array}{l}\text { Son aquellas contribuciones y/o aportes, los cuales presenta todas y cada una de las Partes } \\
\text { en una base de compromisos. }\end{array}$ \\
\hline Mitigación del cambio climático & $\begin{array}{l}\text { Es el principio para reducir, prevenir o eliminar las emisiones de gases de efecto invernadero } \\
\text { de la atmósfera. }\end{array}$ \\
\hline Resiliencia climática & $\begin{array}{l}\text { Según el IPCC es la "capacidad de un sistema social o ecológico para absorber una altera- } \\
\text { ción sin perder ni su estructura básica o sus modos de funcionamiento, ni su capacidad de } \\
\text { auto-organización, ni su capacidad de adaptación al estrés y al cambio" }{ }^{(4)} \text {. }\end{array}$ \\
\hline
\end{tabular}


Artículo 7 del Acuerdo, que busca mejorar la capacidad adaptativa, fortalecer la resiliencia y reducirvulnerabilidad al cambio climático para contribuir al desarrollo sostenible. El Perú podría desarrollar sinergias entre sus sistemas de adaptación y las políticas de salud para disfrutar de cobeneficios en salud. El Acuerdo propone lograrlo a través de la cooperación internacional en los siguientes frentes (22): (i) compartir buenas prácticas y lecciones aprendidas respecto a ciencia, planeamiento e implementación de políticas de adaptación; (ii) mejorar la estructura institucional para apoyar la síntesis de conocimiento, junto con la previsión de apoyo técnico a las Partes; (iii) fortalecer el conocimiento científico sobre el clima, incluyendo investigación del sistema climático y sistemas de alerta temprana; (iv) ayudar a países en vías de desarrollo a identificar medidas de adaptación efectivas, prioridades, desafíos, brechas, y (v) mejorar la efectividad y durabilidad de políticas de adaptación. Afortunadamente para el mundo en vías de desarrollo, bajo el Artículo 9, los países desarrollados se comprometen a proveer recursos financieros con fines de adaptación y mitigación del cambio climático.

A la luz de estos mecanismos, es necesario dilucidar los espacios que tiene el Perú para incluir a las ENT en el futuro Plan Integral de mitigación y adaptación frente a los efectos del cambio climático en la salud pública ${ }^{(20)}$.

\section{RECOMENDACIONES POLÍTICAS DE COBENEFICIOS ENTRE CAMBIO CLIMÁTICO Y ENT}

El Perú es un país vulnerable a los efectos del cambio climático. En este contexto, nace la oportunidad de plantear a los tomadores de decisiones políticas de mitigación y adaptación al cambio climático que presenten efectos positivos sobre las ENT (Tabla 2). Los mejores actores para implementar tales políticas son la Academia y el Gobierno, porque en estos espacios se podrían obtener los resultados más tangibles. La academia cumpliría el rol de generar evidencia en cambio climático y salud; el Gobierno establecería prioridades nacionales de investigación, proveería financiamiento, y propondría, diseñaría e implementaría políticas de salud pública alineadas al Acuerdo de París y el iNDC del Perú.

\section{RECOMENDACIONES PARA LA ACADEMIA}

1. Identificar áreas prioritarias de investigación en la interfaz Clima-ENT y crear capacidades en ellas. La creación de una lista de prioridades de investigación en Clima-ENT garantiza la producción de evidencia para la implementación de políticas de mitigación y adaptación al cambio climático. La priorización señalaría a los stakeholders -investigadores, financiadores y gobierno- a tomar acción en este frente. La priorización debe acompañar a la creación de capacidades, pues la conciencia sobre el efecto en la salud del cambio climático es insuficiente, incluso en futuros profesionales de la salud ${ }^{(23)}$.

2. Generar evidencia sobre la efectividad de intervenciones Clima-ENT. Poca evidencia sobre salud y cambio climático se enfoca en el rol de las ENT. Esta insuficiencia nos impide actuar contra los posibles daños a la salud. La evidencia permitirá evaluar intervenciones que pueden combatir el cambio climático y las ENT sinérgicamente. Debido a su vulnerabilidad, al Perú le urge buscar mecanismos de protección de salud cardiovascular y respiratoria ${ }^{244}$. Esto supliría al discurso técnicopolítico de las herramientas necesarias para tomar medidas de adaptación y mitigación.

3. Anticipar soluciones a la inseguridad alimentaria $y$ estrés hídrico. El cambio climático puede traer graves consecuencias sobre la disponibilidad, acceso y utilización de estos recursos ${ }^{(14)}$, lo que llevaría al consumo de productos de baja calidad y pobre aporte nutricional, causando efectos adversos de ENT a largo plazo. Asimismo, las actividades de la industria alimentaria mundial producen entre el 17 y $32 \%$ de emisiones globales de GEI, lo que está llevando a buscar alternativas que ofrezcan sinergias entre la salud humana y planetaria con

Tabla 2. Recomendaciones para academia y gobierno (plazos estimados de implementación)

\begin{tabular}{|c|c|c|}
\hline Actor & Recomendaciones & Plazo estimado \\
\hline \multirow{3}{*}{ Academia } & Identificar áreas prioritarias de investigación en ENT-CC y crear capacidades en ellas. & Corto \\
\hline & Generar evidencia sobre la efectividad de intervenciones Clima-ENT & Corto \\
\hline & Anticipar soluciones a la inseguridad alimentaria y estrés hídrico previstos & Mediano \\
\hline \multirow{4}{*}{ Gobierno } & Mejorar el planeamiento urbano y adoptar retórica de cobeneficios en salud & Mediano \\
\hline & Invertir en investigación sobre ENT-CC & Mediano \\
\hline & Alinear las agendas de salud y cambio climático & Largo \\
\hline & Desarrollar resiliencia climática dentro del sistema de salud & Largo \\
\hline
\end{tabular}


énfasis en la seguridad alimentaria ${ }^{(25)}$. El Perú puede tomar lecciones sobre las pérdidas durante el fenómeno El Niño de $1997^{(26)}$, actuando como proxy del cambio climático, e investigar soluciones alternativas de agricultura y ganadería ${ }^{(13)}$.

\section{RECOMENDACIONES AL GOBIERNO EN SALUD PÚBLICA}

1. Mejorar el planeamiento urbano y adoptar retórica de cobeneficios en salud. Debido a la rápida urbanización del Perú, debemos asegurarnos que el planeamiento urbano, presente y futuro, sea uno ideal para reducir ENT y emisiones de GEI. Los diseños urbanos municipales deben incluir espacios caminables y ciclovías, pues son cruciales para promover la actividad física, reducir los índices de masa corporal y la contaminación ambiental (27). Adicionalmente, el incremento de áreas verdes favorece un mecanismo de mitigación, pues permite la biosecuestración del $\mathrm{CO}_{2}$; asimismo, crear hubs comunitarios, que incrementen la conectividad urbana, reduce el uso de vehículos motorizados y, por lo tanto, las emisiones de GEl ${ }^{(13)}$. Estas políticas, al reducir tasas de obesidad y diabetes, presentan una oportunidad para introducir argumentos sólidos que giren en torno a cobeneficios en salud ${ }^{(24)}$.

2. Invertir en investigación sobre Clima-ENT. La determinación de prioridades de investigación en la interfaz de cambio climático con ENT abre las puertas a científicos a un nuevo campo de investigación en el Perú. Con ello se requerirán fondos de investigación sobre la efectividad de medidas de adaptación de nuestros sistemas de salud y respuesta a la inseguridad alimentaria y estrés hídrico. Esto permite al Perú usar los fondos climáticos acordados por los países desarrollados en el Acuerdo de París ${ }^{(21)}$.

3. Alinear las agendas de salud y cambio climático. Dentro del diálogo de alto nivel en cambio climático, el Perú creó una Comisión Multisectorial donde se congrega a la Presidencia del Consejo de Ministros para realizar los informes subsecuentes al iNDC ${ }^{(20)}$. Dado al desafío que presentan las ENT y el cambio climático, respuestas políticas y legislativas intersectoriales y unificadas son necesarias ${ }^{(28)}$.
4. Desarrollar resiliencia climática dentro del sistema de salud. Como consecuencia de las medidas adaptativas, la resiliencia climática del Perú va a incrementar. El Sistema Nacional de Salud debería formalizar, bajo su función de protección de la salud, el criterio de resiliencia climática para alinear las metas de salud con ese fin.

\section{CONCLUSIONES}

A pesar de que el Perú se encuentre listado entre los países más vulnerables al cambio climático, se le abre una ventana de oportunidades con el Acuerdo de París para incidir en la interfaz de ENT y cambio climático. La evidencia de la convergencia de ENT y cambio climático que se debe producir generará suficientes argumentos para priorizar a las ENT dentro de la agenda de cambio climático del Perú. Nuestro contexto en transición epidemiológica, junto con las herramientas científicas y financieras del Acuerdo de París, permiten a la comunidad científica y al Estado peruano tomar la delantera en este desafío; ubicarnos como protagonistas en la lucha contra el cambio climático y las ENT entre los países en vías de desarrollo; y convertirnos en referente para los demás países latinoamericanos.

Contribuciones de autoría: JLA, JB, DA y JJM han participado en la concepción del artículo, la revisión crítica y la aprobación de su versión final. JLA, JB y DA contribuyeron con la revisión de la literatura y la redacción del borrador de este manuscrito.

Fuente de financiamiento: JLA, JB y DA han participado de actividades de mentoría, a cargo de JJM, como parte de un proyecto financiado por el Inter-American Institute for Global Change Research (IAI CRN3036). JLA y JJM están afiliados a CRONICAS Centro de Excelencia en Enfermedades Crónicas, Universidad Peruana Cayetano Heredia, el cual recibió apoyo por parte del National Heart, Lung and Blood Institute (NHLBI), National Institute of Health (NIH), Department of Health and Human Services, mediante contrato No. HHSN268200900033C. JJM recibe además financiamiento por parte de Fogarty International Centre (R21TW009982), Grand Challenges Canada (0335-04), International Development Research Center Canada (106887-001), Medical Research Council UK (M007405), National Heart, Lung and Blood Institute (U01HL114180), National Institutes of Mental Health (U19MH098780).

Conflictos de interés: los autores declaran no tener conflicto de interés.

\section{REFERENCIAS BIBLIOGRÁFICAS}

1. PatzJA, Gibbs HK, FoleyJA, RogersJV, Smith KR. Climate change and global health: quantifying a growing ethical crisis. EcoHealth. 2007;4(4):397-405. doi: $10.1007 /$ s10393-007-0141-1
2. World Health Organization. Global action plan for the prevention and control of noncommunicable diseases 2013-2020. Ginebra: WHO; 2013. $103 \mathrm{p}$.
3. A Commission on climate change. Lancet. 2009;373(9676):1659. doi: 10.1016/S0140-6736(09)60922-3.

4. IPCC. Annex II: Glossary. En: Barros VR, Field CB, Dokken DJ, 
Mastrandrea MD, Mach KJ, Bilir TE, et al., editores. Climate Change 2014: Impacts, Adaptation, and Vulnerability Part B: Regional Aspects. Contribution of Working Group II to the Fifth Assessment Report of the Intergovernmental Panel on Climate Change. Cambridge, United Kingdom and New York, USA: Cambridge University Press; 2014. p. 1757-76.

5. IPCC. Climate change 2013: The physical science basis. Contribution of Working Group I to the Fifth Assessment Report of the Intergovernmental Panel on Climate Change Cambridge, United Kingdom and New York, USA: Cambridge University Press; 2014. 1535 p. doi:10.1017/CBO9781107415324

6. Sterk A, Schijven J, de Nijs T, de Roda Husman AM. Direct and indirect effects of climate change on the risk of infection by water-transmitted pathogens. Environmental Sci Technol. 2013;47(22):12648-60. doi: 10.1021/ es403549s

7. Jessup CM, Balbus JM, Christian C, Haque E, Howe SE, Newton SA, et al. Climate change, human health, and biomedical research: analysis of the National Institutes of Health research portfolio. Environ Health Perspect. 2013;121(4):399-404. doi: 10.1289/ ehp. 1104518

8. Grasso M, Manera M, Chiabai A, Markandya A. The health effects of climate change: a survey of recent quantitative research. Int J Environ Res Public Health. 2012;9(5):1523-47. doi: 10.3390/ijerph9051523

9. Poursafa P, Keikha M, Kelishadi R. Systematic review on adverse birth outcomes of climate change. J Res Med Sci. 2015;20(4):397-402.

10. Campbell-Lendrum DH, Corvalan CF, Pruss-Ustün A. How much disease could climate change cause. En: McMichael AJ, Campbell-Lendrum DH, Corvalan CF, Ebi KL, Githeco AK, Scheraga JD, et al., editores. Climate change and human health: risks and responses. Geneva: WHO; 2003. p. 133-158.

11. United Nations, General Assembly. Political declaration of the High-level Meeting of the General Assembly on the Prevention and Control of Noncommunicable Diseases. Geneva: WHO; 2012. p. 13.

12. Horton R. Non-communicable diseases: 2015 to 2025. Lancet.
2013;381(9866):509-10. doi: 10.1016/S0140-6736(13)60100-2

13. Friel S, Bowen K, Campbell-Lendrum D, Frumkin H, McMichael AJ, Rasanathan K. Climate change, noncommunicable diseases, and development: the relationships and common policy opportunities. Annu Rev Public Health. 2011;32:13347. doi: 10.1146/annurevpublhealth-071910-140612

14. Colagiuri R, Boylan S, Morrice E. Research Priorities for NCD Prevention and Climate Change: An International Delphi Survey. Int J Environ Res Public Health. 2015;12(10):12941-57. doi: 10.3390/ ijerph121012941

15. Edwards P, Roberts I. Population adiposity and climate change. Int $\mathrm{J}$ Epidemiol. 2009;38(4):1137-40. doi: 10.1093/ije/dyp 172

16. Daar AS, Singer PA, Persad DL, Pramming SK, Matthews DR, Beaglehole R, et al. Grand challenges in chronic non-communicable diseases. Nature. $\quad$ 2007;450(7169):494-6. doi:10.1038/450494a

17. Beaglehole R, Bonita R, Horton R, Adams C, Alleyne G, Asaria P, et al. Priority actions for the noncommunicable disease crisis. Lancet. 2011;377(9775):1438-47. doi: 10.1016/S0140-6736(11)60393-0

18. United Nations (UN). United Nations Framework Convention on Climate Change. New York; UN; 1993.

19. United Nations Framework Convention on Climate Change (NFCCC). Report of the Conference of the Parties on its twentieth session, held in Lima from 1 to 14 December 2014. Addendum. Part two: Action taken by the Conference of the Parties at its twentieth session. Ginebra: United Nations; 2014.

20. Perú, Ministerio del Ambiente. Contribución Prevista y Determinada a Nivel Nacional (INDC) de la República del Perú. Lima: MINAM; 2015.

21. S. Mayhew, J. Hanefeld. Planning adaptive health systems: the climate challenge. Lancet Glob Health. 2014;2(11):e625-e6. doi: 10.1016/ S2214-109X(14)70313-4

22. Conference of the Parties. Paris Agreement. Paris: 2015.

23. Nigatu AS, Asamoah BO, Kloos H. Knowledge and perceptions about the health impact of climate change among health sciences students in
Ethiopia: a cross-sectional study. BMC Public Health. 2014; 14:587. doi: 10.1186/1471-2458-14-587

24. Watts N, Adger WN, Agnolucci P, Blackstock J, Byass P, Cai W, et al. Health and climate change: policy responses to protect public health. Lancet. 2015;386(10006):1861914. doi: 10.1016/S01406736(15)60854-6

25. Demaio AR, Rockström J. Human and planetary health: towards a common language. Lancet. 2015;386(10007):e36-7. doi: 10.1016/S0140-6736(15)61044-3

26. The season of El Niño [Internet]. The Economist. The Economist Newspaper; 1998 [citado: 20 dic 2015]. Disponible en: http://www. economist.com/node/127009

27. Popkin BM. Global nutrition dynamics: the world is shifting rapidly toward a diet linked with noncommunicable diseases. Am J Clin Nutr. 2006;84(2):289-98.

28. Boylan S, Syrett K, Colagiuri R. Role of law at the non-communicable diseases-climate change interface: considerations for planetary and population health policy. Public Health. 2013;127(6):579-81. doi: 10.1016/j.puhe.2013.01.022

29. Causas del cambio climático [Internet]. World Meteorological Organization. 2016 [citado: 20 dic 2015]. Disponible en: https://www. wmo.int/pages/themes/climate/ causes_of_climate_change.php

30. World Meteorological Organization (WMO). The Global Climate 2001 2010: A Decade of Climate Extremes. Ginebra: WMO; 2013.20 p.

Correspondencia: José Luis Avilez Escribens Dirección: Calle Murcia 125 La Estancia Oeste, La Molina. Lima, Perú. Teléfono: (511) 993772995 Correoelectrónico:jose.avilez.e@upch.pe 